\title{
Evaluación Remota: Consideraciones para Puerto Rico Durante y Después del COVID-19
}

\section{Remote Assessment: Considerations for Puerto Rico During and After COVID-19}

\author{
Oxalis N. Jusino-Aldarondo ${ }^{{ }^{*}}$ \\ 1 Ponce Health Sciences University, Ponce, Puerto Rico. (D) https://orcid.org/0000-0003-4185-7820 \\ * Correspondencia: ojusino@psm.edu
}

Recibido: 16 junio 2020 | Aceptado: 29 junio 2020 | Publicado: 15 julio 2020

WWW.REVISTACARIBENADEPSICOLOGIA.COM

\section{Citar como:}

Jusino-Aldarondo, O. (2020). Evaluación remota: Consideraciones para Puerto Rico durante y después del COVID-19. Revista Caribeña de Psicología, 4(2), 166-175. https://doi.org/10.37226/rcp.v4i2.4855

\section{RESUMEN}

En la época de COVID-19 muchos psicólogos puertorriqueños tuvieron que reinventarse con el uso de la tecnología para poder ofrecer sus servicios. En el artículo se revisa y analiza la literatura histórica sobre la evaluación remota y cómo ha evolucionado. Del mismo modo, resalta la importancia de proporcionar una comprensión del estado actual de esta modalidad antes de comenzar a ejecutar esta práctica. Para poder hacer uso de la evaluación remota es importante conocer sus implicaciones éticas, sus modalidades y el debido proceso de administración. Se discuten las leyes actuales en Puerto Rico y las recomendaciones de varias asociaciones de psicología respecto al tema. Además, se revisan los materiales necesarios como cámaras, monitores y plataformas en línea. También se analizan medidas psicológicas que evidencian la utilización de la evaluación remota y cómo se pueden ajustar para satisfacer necesidades particulares. Algunas pruebas de ejecución, como la Reynold's Adaptable Intelligence Test, Weschler Intelligence Scales for Children $V$ y la Woodcock Johnson IV, se han estandarizado para su administración de forma remota o se han realizado estudios equivalentes. Se considera cómo el periodo de cuarentena debido al COVID-19 puede afectar de alguna manera a la enseñanza y supervisión del proceso de evaluación y las competencias profesionales. Por último, recomendaciones de cómo se podría utilizar la evaluación remota en el futuro en Puerto Rico.

Palabras Claves: COVID-19; evaluación psicológica; tele-evaluación; tele-medicina

\begin{abstract}
In the times of COVID-19 many Puerto Rican psychologists had to reinvent themselves with the use of technology to be able to offer their services. This article reviews and discusses historical literature on remote evaluation and how it has evolved. Also, provides an understanding of the current status of this modality before beginning this practice. To be able to make use of remote evaluation it is important to know the ethical implications, its modalities and the process of how to carry it out. Therefore, the current laws in Puerto Rico and the recommendations of various psychology associations are discussed. Also, some of the necessary materials are reviewed such as cameras, monitors and online platforms. In addition, the psychological measures that have evidence that remote evaluation could be used, are analyzed, and how some can be adjusted to meet the need. Some performance tests like the Reynold's Adaptable Intelligence Test, Weschler Intelligence Scales for Children V y Woodcock Johnson IV (RAIT,
\end{abstract}


WISC-V and WJ-IV) have been remotely standardized or equivalent studies have been conducted. Furthermore, it is considered how COVID-19 can somehow affect the teaching and supervision of the assessment processes and competences. Finally, recommendations of how remote assessment could be used in the future in Puerto Rico.

Keywords: COVID-19; psychological assessment; tele-assessment; tele-medicine

\section{INTRODUCCIÓN}

Con la llegada del COVID-19 a Puerto Rico, llegó la incertidumbre a muchos profesionales de la salud mental, afectando de manera particular a los psicólogos que especializan su práctica en la evaluación. Esto debido a que es una de las áreas primordiales del ejercicio de los especialistas en conducta humana en nuestra isla (APPR, 2011). ¿Cómo podemos evaluar bien usando medios electrónicos? Fue una de las preguntas que muchos se hicieron. Este artículo discute las posibilidades de cómo practicar este modo de evaluación de manera adecuada.

El COVID-19 es una enfermedad de carácter respiratorio que fue declarada pandemia por la Organización Mundial de la Salud (OMS) el 11 de marzo de 2020. Esa misma semana ya se reportaban los primeros casos en Puerto Rico, y para el 15 de marzo se firmó la primera orden ejecutiva (OE-2020-023), que declaraba la isla en un estado de emergencia. En dicha orden, se estableció un toque de queda y el cierre de la mayor parte de la actividad económica. Esto provocó que muchos especialistas de la salud mental se quedaran sin recursos viables para atender a sus pacientes y comenzaron a buscar opciones alternas para segur brindándoles un servicio seguro, confidencial y privado.

La Junta Examinadora de Psicólogos, actuó de manera expedita y publicó una resolución en donde permitía el uso de la tecnología para ofrecer servicios a distancia a partir del 14 de marzo de 2020 (Junta Examinadora de Psicólogos de Puerto Rico, 2020). En la misma, se recomienda que todo el que vaya a hacer uso de la tecnología, debe tener conocimiento especializado y discutir el procedimiento de evaluación remota a través de un consentimiento firmado que asegure una práctica ética y competente en el uso de dicha modalidad.

El 20 de marzo, se flexibiliza la Ley Núm. 168 (2018), “Ley para el Uso de la Telemedicina en Puerto Rico" bajo la Resolución Conjunta 641. Luego, se creó la Ley Núm. 48 (2020) “Ley para Regular la Ciber- terapia en Puerto Rico". En esta se incluyen a los estudiantes del programa de Educación Especial del Departamento de Educación. Dichas leyes permitieron que muchos psicólogos pudieran ofrecer sus servicios de manera segura y que los planes médicos pagaran por los mismos.

Sin embargo, aún queda un trayecto por cubrir. El área de la evaluación sigue siendo un reto de amplios obstáculos por superar. La ley no hace mención del área de evaluación, siendo esta una de las más importantes para el diagnóstico, registro y tratamiento de los menores que participan del programa de Educación Especial, además de un sin número de procedimientos de salud y forenses que requieren algún tipo de evaluación. En cuanto al área de evaluaciones a menores en edad escolar, la Asociación de Psicología Escolar (2020) se expresó en contra de cualquier evaluación hecha en modalidad remota. Por otra parte, recomendaron el uso de instrumentos de cernimientos que tuvieran urgencia o que fueran necesarios, y que toda evaluación comprensiva se atrase hasta poder hacerla de manera presencial. Además, le sugieren a las escuelas privadas no pedir evaluaciones de admisión. Esta comunicación sentó las bases para que no se hicieran evaluaciones remotas en la isla. Sin embargo, la misma no es ley, por lo cual deja la puerta abierta a que profesionales adiestrados en el área puedan realizar esta práctica a su discreción.

Cabe señalar, que el área de evaluación por la vía remota implica más retos en su práctica que la terapia. Esto debido a una variedad de factores. Entre estos, el ofrecer una evaluación de manera confidencial sin poner en riesgo la seguridad, validez y confiabilidad de la prueba, así como poder hacer y documentar observaciones pertinentes y claras sobre la persona evaluada y su proceso evaluativo. Al respecto, este artículo expondrá los antecedentes de la evaluación remota, su presente las recomendaciones para una práctica ética, modelos, procedimientos, pruebas a utilizar, enseñanza, supervisión y finalmente analizar la situación actual en Puerto Rico. 


\section{ANTECEDENTES DE LA EVALUACIÓN REMOTA}

La evaluación remota no es algo novel, se pretende comprender sus principios para poder entender cómo funciona. Para los años 80s, se comenzó a investigar cómo el uso de la computadora se podía viabilizar en la evaluación psicológica. Uno de los primeros estudios sobre el uso de computadores conectadas a internet fue el de Byers (1981), donde plantea que usar una computadora para hacer evaluaciones disminuye su costo por la reducción en el tiempo de corrección e interpretación. En esa investigación se administró la Minnesota Multiphasic Personality Inventory (MMPI) a través de la computadora, sin embargo, Byers aclara que solo se debe usar para tamizajes. Cabe destacar, que el método empleado para su investigación no se discutió.

Glaze y Cox (1991) validaron una escala computarizada de depresión postnatal. Se administró en ambos formatos, papel y lápiz y computadora. No encontraron diferencias en la manera que se llenaba el cuestionario, que era completado por las mujeres en una oficina dentro de una clínica. Aunque este no es un estudio de evaluación remota per se, ya se comenzaban a sentar las bases para el envío de cuestionarios en línea. En 1998, Ball y Puffett investigaron la viabilidad de usar equipo de videoconferencia para evaluar la función cognitiva en la población geriátrica. Determinaron que la prueba Cambridge Cognition Examination (CAMCOG) puede ser usada de manera confiable a través de una plataforma de videoconferencia. Dicha investigación se llevó a cabo usando un ayudante al lado de la persona evaluada.

Luego, en 1999, Ball y colegas investigaron la administración del Mini Mental Status Exam (MMSE) usando videoconferencia. Encontraron que no había diferencias cuando se administraba por vídeo, sin embargo, encontraron dificultad para poder corregir de manera adecuada la oración del instrumento. Asimismo, argumentaron que esperaban que los avances tecnológicos sobrepondrían las limitaciones que enfrentaron y ofrecieron la opción de poder corregirlo de manera presencial luego de ser administrado. En el 2000, Maheu y Gordon, expusieron una nomenclatura específica para la evaluación en línea Evaluación Psicológica Basada en la Web (Web-Based Psychological Assessment). Actualmente, esto es solo parte de lo que puede ser la evaluación remota. Por otro lado, Vanden Bos y Williams (2000) encontraron que un 15\% psicólogos/as encuestados utilizaban medios como email, fax y otros, mientras que un $11 \%$ lo hacía por el teléfono, para evaluaciones psicológicas y neuropsicológicas. A su vez, Maheu y Gordon (2000) encontraron que un $68 \%$ de los que practicaban en línea ejecutaban la evaluación basada en la web, en donde la persona iba a una página de internet y completaba un cuestionario o ejecutaba la prueba. Sin embargo, estos estudios no especifican las pruebas utilizadas por dichos especialistas. Kirkwood (2000) administró de manera remota varias pruebas como la National Adult Reading Test (NART), su versión Quick Test y el Adult Memory and Information Processing Battery (AMIPB) y encontró que al ser administradas de manera remota daban resultados similares a las presenciales. Por otra parte, una de las dificultades que exponen fue la comprensión ortográfica de las respuestas escritas de las personas evaluadas. Muchos de estos estudios se enfocaban en cuestionarios puestos en la Web y no eran de uso clínico exclusivo necesariamente, sino que podían ser usados por cualquiera, sin la necesidad de ser licenciado en el área de salud mental.

Sin embargo, Buchanan (2002) discute que los instrumentos que estaban disponibles hasta ese momento (cuestionarios en su mayoría), fueran usados solamente bajo dos condiciones: 1) investigación, en donde los resultados de dichas pruebas no afectarían directamente a la persona evaluada y 2) en el contexto de terapia o consejería en línea, donde el profesional pudiera discutir los resultados y ofrecer consejería de los mismos. Entre las desventajas y posibles problemáticas discutidas por Buchanan y Barak (2004) se encuentran el que haya problemas con la conexión y el riesgo a que la comunicación sea interceptada por un tercero. Además, una evaluación de manera anónima no le permitiría al profesional el saber a quién evalúa. Otra desventaja es la normativa utilizada para comparar al individuo. Añaden que en evaluaciones remotas no hay control del ambiente evaluativo (que puede estar lleno de distractores) y que, además, hay personas que responden de manera diferente al ser inhibidos por la tecnología. La última desventaja que señalan es la necesidad de controles y regulaciones, que para aquel entonces eran menos que en el presente. 
Estos mismos autores mencionan algunas ventajas de la evaluación remota. Entre estas, la flexibilidad que tiene la persona de completar la evaluación a su tiempo. También, al ser corregidas de manera digital hay menos error humano en los cálculos. Además, el especialista estaría menos tiempo corrigiendo instrumentos. El evaluar de manera digital, provee la capacidad de siempre tener accesible la versión más actualizada del instrumento. Por último, mencionan que las limitaciones del distanciamiento físico son acortadas a través del uso de la tecnología. Naglieri y colegas, (2004) exponen que en la evaluación remota se mantienen los mismos problemas que la evaluación presencial. Plantean que la evaluación remota, tiene ciertas ventajas y desventajas, previamente discutidas (Buchanan \& Barak, 2004). Por su parte, estos autores analizaron detalladamente las implicaciones éticas de la evaluación a distancia como, por ejemplo, la seguridad de las pruebas y la integridad de la representación de las personas evaluadas. Hacen un análisis acerca de las preocupaciones éticas sobre cómo el alterar un proceso estandarizado se compromete la confiabilidad de los resultados. Además, discuten los retos de evaluar a personas con discapacidad y a minorías, entre las que podría estar el contexto puertorriqueño.

Brearly y colegas (2017) hicieron un metaanálisis de investigaciones sobre evaluación remota. En el mismo, se incluyeron 12 estudios de corte neuropsicológico, que estaban en el rango de años entre el 1997 hasta el 2016. La muestra utilizada fue de 497 participantes a través de las 12 investigaciones. Dentro de los hallazgos obtenidos, se destaca que no encontraron diferencias significativas entre los resultados de evaluaciones remotas y presenciales.

\section{PRÁCTICAS ADECUADAS}

Muchos de los estudios ya citados abrieron paso a lo que hoy día se conoce como evaluación remota. Luxton y colegas (2014) describieron las mejores prácticas para la evaluación remota. Estas son las siguientes: 1) la evaluación se debe dar en un ambiente y momento que sea cómodo y sin interrupciones, 2) guiar a la persona en el uso apropiado de la tecnología; específicamente la ubicación de las cámaras, 3) una conexión de internet adecuada para el procedimiento, 4) considerar el manejo y habilidad en el uso de la tecnología y 5) aceptación de la modalidad de evaluación por la persona a ser evaluada.

Hay que considerar la disponibilidad de materiales en el hogar; incluyendo la posición frente a la cámara de la persona a evaluar. Cada uno de los puntos expuestos acarrea asuntos indispensables a considerar al momento de evaluar. Para abundar sobre el tema, discutiremos de manera detenida el punto del uso apropiado de la tecnología. Es importante que la persona evaluada o la identificada como asistente en la evaluación, sepa colocar las cámaras y se dirija, remotamente, a la misma. Esto se debe a que la ubicación de las cámaras permitirá poder observar a la persona y su trabajo, de manera adecuada. El contacto visual directo se verá afectado por la tecnología por lo que se debe procurar, a medida de lo posible, disminuir esta barrera. Además, hay que asegurarse de que la persona tenga una conexión a internet adecuada para poder hacer el procedimiento. Esto se puede hacer antes de comenzar, realizando una prueba para verificar la estabilidad de la conexión y/o enviando un cuestionario a ser completado acerca del tipo de conexión disponible en el hogar y qué cosas puede o no hacer (ej. ver películas, jugar videojuegos online). También se debe indagar el grado de alfabetización digital y tenerlo en consideración antes de programar una evaluación remota (Luzton et al., 2014).

Otro factor a considerar es el consentimiento de la persona a ser evaluada de manera remota y que esté consciente de las implicaciones (beneficios y riesgos) que esto puede conllevar. Usualmente la mayoría acepta a esta modalidad evaluativa, pero es importante tener hojas de consentimiento específico para estas tareas y hojas explicativas con el proceso a seguir. Asimismo, es imperativo solicitar contactos telefónicos al evaluado y tenerlos disponibles en caso de que ocurra alguna situación de emergencia. Hay que, además, tener en cuenta los antecedentes culturales de la persona a evaluar. En Puerto Rico, se tendría que estar pendiente a las variables sociodemográficas de la persona para poder ser sensibles en el proceso evaluativo. Otra práctica adecuada en la evaluación remota es el poder hacerla de manera ética y con los estándares de privacidad esperados en una evaluación presencial (Luxton et al., 2014). 
La Asociación Americana de Psicología (APA, por sus siglas en inglés) publicó seis principios para la evaluación remota en donde: el primero, de ellos es velar por la seguridad de la prueba y el mantener los estándares éticos de la evaluación (APA, 2020). En cuanto a la seguridad de las pruebas, se han hecho esfuerzos por parte de todas las casas publicadoras para que el material esté disponible en línea y no tenga que enviarse por correo. Sin embargo, una de las prácticas comunes en la evaluación remota es enviar protocolos y algunos estímulos al hogar de la persona evaluada (Wright, 2020). Esto trae muchísimas dudas en cuanto a cómo se manejan dichos documentos y/o estímulos, pues puede comprometer la integridad del instrumento de evaluación. El envío de protocolos, debe hacerse a través de un sobre sellado con una estampilla firmada en el borde de la apertura, para que el mismo sea abierto en cámara justo antes de comenzar la evaluación. Al finalizar la cumplimentación de los protocolos enviados se sellan de manera similar, en cámara. El segundo principio, a discutir, es que se recomienda el expandir los intervalos de confianza. Aunque, no hay una dirección clara de cuánto hay que expandirlos.

Durante un webinario, el Dr. Jordan Wright (2020) indicó que él estaba seguro que probablemente se podía suponer que la ejecución del menor se inclinaría más hacia una mejor puntuación. Sin embargo, expuso que había que estipular las puntuaciones usando intervalos de confianza más amplios. El tercer principio, es el poder pensar críticamente sobre las sustituciones de subpruebas y de pruebas. Debido a que no todas las pruebas y/o subpruebas van a poder administrarse de manera remota; específicamente, durante el transcurso de tiempo de la crisis. Es por eso que como especialistas en el área de evaluación debemos hacer un análisis exhaustivo y detenido en cuanto a qué pruebas escoger, y cuáles sustituciones son posibles. Sobre esto, y con el fin de ejemplificar, la WISC V no permite sustituir todas las subpruebas en sus respectivos índices, la prueba provee la posibilidad de una sola sustitución para poder calcular el Cociente Intelectual Total (CIT). El cuarto principio a mencionar, es tener en cuenta la calidad de los datos. No necesariamente los datos que se obtengan de manera remota serán igual de válidos y confiables que cuando se administra presencialmente. Se debe considerar la posibilidad de que hayan más distractores o que la comunicación se interrumpa en medio de una prueba de velocidad de procesamiento. Por lo cual, es importante documentar todo y tener en cuenta toda esta información al momento de interpretar.

El quinto principio es el hacer lo mejor que puedas con lo que tienes, ética y conscientemente. Esto especialmente, durante la emergencia. Cabe señalar que la recomendación de la APA y el Dr. Wright, es de esperar al momento adecuado para evaluar, de no ser urgente, para así hacerla cuando haya culminado la emergencia y se pueda hacer presencial. APA (2020a), en otra publicación, indican que la administración de pruebas de funcionamiento se hace de forma estandarizada, por lo cual podría ser la que más se afecte significativamente por la modalidad de evaluación remota. El ultimo principio, es mantener los mismos estándares éticos de cuidado que cuando evaluamos de manera presencial. Se enfatiza en que haya un consentimiento informado que sea claro y especifico al evaluar de manera remota.

Con respecto al tema de enseñanza y supervisión, la APA propone alternativas para los evaluadores a través de unas útiles guías recientemente publicadas (APA 2020b). Dentro de estas, se exhorta a considerar la pertinencia de hacer o no evaluaciones en estos momentos; sugerencia que coincide con los planteamientos de otras asociaciones mencionadas anteriormente. Cada caso es único y tiene sus particularidades; es responsabilidad del profesional determinar si la evaluación es de carácter de urgencia o no. En algunos casos, se tendrán que hacer referidos especiales a profesionales con la pericia apropiada para llevar a cabo una evaluación que se ajuste a las necesidades del individuo. Asimismo, se recomienda el hacer evaluaciones más limitadas con instrumentos que tal vez no necesiten grandes alteraciones (APA, 2020).

\section{MODELOS DE EVALUACIÓN REMOTA}

No toda la evaluación remota se administra de la misma manera. Sobre esto, Stolwy y colegas (2020) establecen tres modelos diferentes a seguir. El primero es tener un asistente en una clínica remota mientras el evaluador se encuentra en otra. Este modelo se ha utilizado cuando hay grandes distancias entre especialista y evaluado (Stolwyk et al., 2020). El problema de aplicación de este modelo es que no es posible llevarlo a cabo en Puerto Rico, dado que no existen psicómetras $\mathrm{u}$ otras profesiones similares que puedan 
brindar asistencia en las evaluaciones psicológicas. Tal vez, esa tarea la pudieran llevar a cabo estudiantes en entrenamiento, planteamiento aún inexplorado en Puerto Rico pero que podría enriquecer el proceso de formación de futuros psicólogos evaluadores. En algunos lugares entrenan a maestros para estos propósitos, pero habría que analizar los componentes legales implicados en esta práctica. En los Estados Unidos este modelo puede traer desventajas, ya que el especialista debe asegurarse de que su licencia le permite ejercer fuera de su jurisdicción. Sin embargo, en Puerto Rico las distancias no interfieren tanto como en Estados Unidos.

El segundo modelo, es uno donde especialista y evaluado se encuentran en la misma clínica, pero en oficinas separadas. Esta modalidad, puede garantizar la seguridad de las pruebas, debido a que las mismas no salen de la clínica. Además, se asegura la calidad del internet y se reduce la posibilidad de contagio en tiempos de COVID-19. Este modelo provee la ventaja de no tener que usar mascarilla mientras se evalúa, y se pueden hacer mejores observaciones de expresiones faciales y en supbruebas que requieran el uso de dicción. Si ocurre alguna situación imprevista, el especialista puede intervenir de ser necesario. Además, el evaluador tiene total control del equipo y de la ubicación de las cámaras, computadoras, etc. (Stolwyk et al., 2020)

El último modelo es el más flexible debido a que es la evaluación que se completa desde la casa. En este no hay control de la calidad del internet, se tiene que entrenar a la persona y/o la familia para poder ubicar el equipo de manera adecuada. Si surgiera alguna situación imprevista, el especialista debe tener números a llamar y/o instrucciones a seguir de antemano. Además, este modelo plantea algunos retos para mantener las pruebas seguras, debido a que algunos reactivos habría que enviarlos o simplemente no administrar algunas subpruebas (Stolwyk et al., 2020).

\section{PROCESO EVALUATIVO}

Es importante que antes de comenzar el proceso se firmen todos los consentimientos necesarios. Un ejemplo de este consentimiento se puede encontrar en las guías sobre evaluación y adiestramiento publicadas por la APA (2020a). Entre los procedimientos a llevar a cabo antes de comenzar la evaluación, se recomienda que se completen cuestionarios para eva- luar la posibilidad de recibir el servicio desde la casa, en caso de considerar esta modalidad. Es necesario que se provean números de teléfonos de contactos a los que recurrir en situaciones de emergencia o crisis. También se deben proveer instrucciones sobre cómo situar el equipo a usarse y como posicionarse para la evaluación. Como ya mencionado anteriormente, se pueden enviar materiales a usarse, incluyendo protocolos y/o reactivos.

Una vez discutido el formato, confidencialidad y consentimiento, se puede comenzar la evaluación. Para poder llevar a cabo la misma harán falta varios materiales. Primero, hay que asegurarse que la prueba está disponible para uso en línea. La mayoría de las casas publicadoras han permitido acceso a las pruebas de manera remota y digital. Hay que escoger una plataforma de videollamada que sea apropiada para evaluar y que cumpla con los estándares de la Ley Federal de privacidad de información de salud (HIPAA, por sus siglas en inglés, 1996). Del lado de la persona evaluada, serán necesarias por lo menos dos cámaras, usualmente una en la computadora y otra por detrás de la persona, que en este caso podría ser un teléfono inteligente en modo avión (Sharp, 2020a,b; Wright, 2018).

En el lado del especialista debe haber dos monitores y al menos una cámara. De esta manera puede ver en el monitor más grande las cámaras de la persona evaluada y en el otro manejar los estímulos de la prueba. Si fuera necesario enseñar algo a través de la cámara, se debe acercar lo más posible para que la otra persona lo pueda ver. Por esto es importante el tener todos los materiales de la prueba accesibles al momento de evaluar. A su vez, se entiende que el tener más de un protocolo disponible al momento de evaluar es mejor (Sharp, 2020a,b; Wright, 2018).

\section{INSTRUMENTOS EVALUATIVOS}

Es importante conocer qué instrumentos pueden ser usados apropiadamente de manera remota. A continuación, se discutirán algunos de los instrumentos que han sido investigados, tomando en consideración que en Puerto Rico los instrumentos en español son limitados.

Las plataformas de cada casa publicadora llevan años ofreciendo pruebas para administración remota. Sin embargo, se considera que tienen menos impacto 
al ser administradas de manera remota (APA, 2020b). Por ejemplo, la plataforma de Pearson, Q-Global, ofrece las opciones para evaluación remota de la mayoría de sus cuestionarios. Algunos de estos pueden ser utilizados en Puerto Rico, como el Behavior Assessment System for Children- 3rd Edition (BASC-3) publicada en el 2015 y el Vineland Adaptive Behavior Scales3rd edition, publicada en el 2016. Esta plataforma, además, ofrece la opción de poder administrar la prueba en la oficina en vivo. Así pues, el especialista puede escoger entre enviarla por correo electrónico y que la persona la conteste por videollamada o proyectarla en su pantalla mientras la persona indica o selecciona la respuesta. Otros ejemplos de sistema de evaluación remota, se encuentran en la plataforma de MultiHealth Systems Inc. (MHS), (llamada Assess+), donde se pueden encontrar cuestionarios como el Comprehensive Executive Function Inventory (CEFI, por sus siglas en ingles) y la Conners en su tercera edición.

Por otra parte, la Minnesota Multiphasic Personality Inventory-2 (MMPI-2) no estaba disponible para ser administrada remotamente. Sin embargo, existen guías para una evaluación remota apropiada usando dicha prueba (Corey \& Ben-Porath, 2020). Estos autores publicaron, también, una lista de cotejo con recomendaciones para garantizar la mayor validez posible. Incluso, hacen la salvedad para casos de evaluación en el área forense, donde evaluar de manera remota podría traer muchas complicaciones. Los autores enfatizan en que el manual de la prueba es explícito sobre el momento que una persona está siendo evaluada con la MMPI-2; esta debe ser observada, usualmente por el especialista (en el caso de Puerto Rico). Esta especificación se da debido a que en Estados Unidos se pueden usar psicómetras u otros especialistas para ayudar al clínico. Sin embargo, en Puerto Rico no es posible debido a que la Ley 96 (1983) y el Código de Ética (1992) lo prohíben. Por tanto, hacen hincapié en que de manera remota la prueba también debe ser observada usando una plataforma de videollamada. Debido a la pandemia, Pearson (propietario de los permisos de publicación de la prueba), ha permitido que la misma esté disponible para evaluación remota.

Otra prueba que puede ser útil para usarla de manera remota es la Reynolds Adaptable Intelligence Test (RAIT) y su prueba hermana, Test of General Reasoning Ability (TOGRA). Estas pruebas fueron estandariza- das para su uso tanto en modo presencial como remoto (Reynolds, 2014). Esta prueba está disponible en la plataforma PARiConnect, de la casa publicadora PAR Inc. La prueba cubre las edades de 10 a 75 años y toma alrededor de 50 minutos administrar la batería completa. Hay una versión bilingüe de la prueba, llamada Reynolds Intellectual Assessment Scales-2nd Edition (RIAS-2), para la cual hay una investigación de equivalencia entre la evaluación remota y la presencial (Wright, 2018). Aunque se encontró que las formas eran equivalentes, hubo diferencias al administrar la subprueba de velocidad de procesamiento en niños de seis años o menos, por lo que se recomienda que dicha subprueba no se administre a menores de 6 años. Esta prueba tiene un rango de edad que va desde los tres a los 94 años (Reynolds \& Kamphaus, 2015) y es más corta que la RAIT, ya que toma alrededor de 20 a 25 minutos completarla. Sin embargo, ninguna de estas pruebas tiene normas para Puerto Rico y no están disponibles en español.

Otra prueba que ha mostrado equivalencias al ser administrada de manera remota es la Wechsler Intelligence Scale for Children-Fifth Edition (WISC-V). Para probarlo, se realizó un estudio en Australia, donde encontraron coeficientes de correlación excelentes al comparar la administración en vivo y la remota. La evaluación se ejecutaba en centros específicos, y el menor estaba acompañado de un psicólogo. En este estudio no se excluyeron las subpruebas con estímulos motores, sino que se añadieron cámaras para poder observar el proceso de solución de problemas empleado por el menor. Cabe señalar, que esta ha sido una de las grandes limitaciones de la evaluación remota, sin embargo, este estudio logró sobreponer estas barreras. Incluso, los autores midieron la satisfacción de los padres y de los psicólogos que asistían a los evaluados y encontraron que había una satisfacción excelente y que el modo de administración no afectaba el funcionamiento (Hodge et al, 2018). Todos los libros de estímulos fueron publicados a través de la plataforma de Q-Global, sin embargo, no se incluyó la versión en español. Aunque en comunicación personal con la Sra. Elizabeth Werner, representante para Puerto Rico de Pearson, en el mes de junio de 2020 mencionó lo siguiente:

"Aún no han llegado ahí. Pero se encuentran entre el lote de productos que se priorizan para la siguiente carga de activos por lotes. iNo tengo una fecha exacta 
para ti, pero espero que el conocimiento de que los activos están por llegar ayude en algo!"

En podcast con el Dr. Jeremy Sharp (2020), la Dra. Susan E. Raiford quien es Directora Senior de Investigación de Pearson, explicó que la WISC-V Integrada era una opción para la evaluación remota en el hogar. Explicó que esta prueba presenta la tarea de bloques en selección múltiples y que se pueden sustituir diferentes subpruebas por otras, para calcular índices no motores. Estos índices usan la combinación de la WISC-V junto con una subprueba de la Integrada e incluyen, además, el Índice Total Nomotor, el Índice Noverbal Nomotor, el Índice Nomotor de Habilidad General y el Índice Nomotor Visoespacial. Hay un programa especializado WISC-V Integrated Interpretative Assistant 1.0 que ayuda a calcular estas puntuaciones compuestas (Raiford, 2020), basado en Excel y disponible a través de la plataforma de Q-Global, mientras dure la emergencia por COVID-19. Es importante señalar que una de las subpruebas que se debe administrar es Naming Speed Quantity (Velocidad de Nombramiento de Cantidades) y está disponible solamente en las subpruebas complementarias en inglés.

Por último, mencionaremos la Woodcock-Johnson $I V$, que en su versión en español se conoce como Batería IV. Wright (2018) hizo un estudio de equivalencia entre evaluación remota y presencial, en la cual se encontró que no hay diferencias significativas en la manera de administrar la batería. El estudio se llevó a cabo con niños y niñas entre las edades de cinco a 16 años, por lo cual no hay medidas para adultos; aunque la prueba provee para evaluación con adultos. El mismo autor indicó que se debe colectar más data para validar dicho estudio. La Batería IV está disponible actualmente de manera digital a través de la plataforma de Riverside Insights, Presence Learning, mientras dure la emergencia por COVID-19.

\section{CONSIDERACIONES PEDAGÓGICAS Y SUPERVISIÓN}

Según las guías para la enseñanza y supervisión de evaluaciones publicadas por la APA en junio de 2020, es sumamente importante que se le dé un mayor seguimiento a corto y largo plazo a los estudiantes en el área de evaluación debido a que probablemente tengan más incertidumbre, preguntas y áreas a mejorar que antes. Cuando estemos en un proceso de supervisión, hay que reentrenar y practicar las destrezas que serán necesarias para evaluación remota o algún modelo híbrido de esta. Es importante tomar en consideración el nivel de comodidad del practicante.

En el salón de clase, ya sea virtual, presencial o de manera híbrida, hay que enfatizar en los procedimientos estandarizados de las pruebas. La enseñanza no debe cambiar del todo, hay que continuar enseñando los puntos de comienzo, bases, topes, etc. y añadir las maneras virtuales a través de videos o administraciones con juego de roles. En casos de práctica se podría considerar el poder grabar la sesión a través de la plataforma de videollamada. Se recomienda que se enfoque la administración en lo básico y cubrir a plenitud las bases de una buena administración estandarizada. Las guías resaltan que mientras la evaluación se realice a un voluntario no hay grandes riesgos y se podrían corregir los errores después de haber culminado la evaluación, a diferencia de hacerlo con clientes reales, donde el riesgo es mayor. Por último, se enfatiza en el documento la habilidad del docente a ser flexible ante las necesidades de los estudiantes y supervisados. Incluso, habrá que respetar el hecho de que algunos no se sentirán cómodos a asistir de manera presencial.

\section{CONCLUSIÓN Y RECOMENDACIONES}

En conclusión, la evaluación remota en tiempos de COVID-19 puede ser una alternativa en casos específicos. Por ejemplo, algún paciente que la requiera para tomar alguna decisión urgente, o menores de edad con necesidades especiales que necesiten una evaluación para poder recibir ciertos servicios, porque de otra manera su desarrollo saludable se podría ver afectado. Además de estas condiciones, el atraso en evaluaciones puede provocar la acumulación de casos para los especialistas, y al retomar su práctica de manera presencial tener una cantidad mayor a la que acostumbran. Similarmente, muchos psicólogos generan sus ingresos evaluando y el no poder hacerlo podría poner en peligro sus prácticas y su porvenir.

Una desventaja para Puerto Rico, es que muchas de las pruebas que están disponibles para los usuarios en inglés, para los hispanohablantes no están disponibles. En pruebas de ejecución, podrían ser usadas la RIAS con personas bilingües y la Batería IV cognitiva en español. Ninguna de estas pruebas tiene 
normas para la población puertorriqueña, siendo uno de los mayores problemas en la práctica profesional en la isla. Próximamente, si en efecto, Pearson incluye la versión en español de la WISC-V, tendríamos entonces más opciones para usar.

Otra desventaja, general de la evaluación remota es que se podría perder el análisis de proceso que muchas veces se hace al evaluar. Esto debido a que una o dos cámaras no van a permitir que se pueda observar todo el campo visual que se observa cuando la persona está de frente a uno. También, si se usan pruebas que no tienen el componente motor, se pierden por completo estas observaciones. Sería recomendable, entonces, que de alguna manera la cámara que está por detrás de la persona evaluada también apunte hacia el espacio de trabajo. De esta manera se podría compensar por lo que se pierde en el campo visual del especialista.

Este escrito tuvo su enfoque principal en pruebas de ejecución, pero muchas otras medidas de personalidad como la Rorschach, han sido analizadas para su inclusión en la evaluación remota y sus autores han publicado medidas éticas y adecuadas para su correcta administración por la vía remota (R-PAS, 2020). Cada casa publicadora y cada asociación profesional de psicología local tiene sus guías y materiales para evaluación remota. Es deber de todo profesional estar educado sobre las leyes que rigen la práctica en su jurisdicción, así como ser responsable de investigar las guías de cada prueba que quiera administrar. Ante todo, siempre se debe recordar que, si se puede esperar, es mejorar esperar.

Ahora que estamos al inicio del proceso de reapertura a las actividades cotidianas con menos restricciones de distanciamiento físico y social debido al COVID-19, podríamos considerar el evaluar remotamente, pero en la misma oficina. Esto siendo posible solamente cuando se tiene el equipo necesario y

\section{REFERENCIAS}

American Psychological Association. (2020a). Teaching and supervising assessment beyond COVID19. https://www.apa.org/topics/covid-19/teaching-supervising-assessment-beyond.pdf.

American Psychological Association. (2020b). Guidance on psychological tele-assessment during the COVID-19 crisis. https://www.apaservices.org/practice/reimbursement/healthcodes/testing/tele-assessment-covid-19.

Asociación de Psicología de Puerto Rico. (2011). Aspectos éticos y tomando en consideración su costo-efectividad. Muchos han retomado sus prácticas de evaluación usando mascarillas, pantallas de acrílico y caretas de plástico. Estos procedimientos tampoco están dentro de la estandarización de las pruebas, por lo cual pueden tener un impacto en la ejecución de la persona. Cabe señalar, que todo procedimiento que altere lo establecido por la estandarización debe estar estipulado en el informe de manera clara y explicar por qué se hizo la alteración. En la conclusión de dicho informe debe dejar claro que el proceso es uno comprensivo y que no se toman decisiones con una sola prueba, sino que se toman en consideración todas las variables evaluadas (motivo de referido, observaciones clínicas, e historial, además de las pruebas).

En un futuro cercano, cuando la situación de emergencia culmine, será importante indagar sobre la posibilidad de que en Puerto Rico se pueda establecer una práctica de evaluación remota. Sobre esto, habría que investigar los costos que podría tener este tipo de evaluación para los psicólogos de Puerto Rico, además de investigar cuán dispuestos estarían los puertorriqueños a ser evaluados de esta manera. Esta modalidad podría ser útil para evaluar a personas en Vieques y Culebra y tal vez personas que tengan problemas de transportación o se encuentren lejos del especialista. Se podría utilizar el modelo de ir a una clínica cercana que cuente con todo el equipo necesario, incluyendo internet de banda ancha. Con los debidos procesos de ley, se podrían evaluar boricuas viviendo en otras partes del mundo que tal vez no tengan acceso a los instrumentos y normas de Puerto Rico. Tenemos un campo nuevo para explorar en la psicología de Puerto Rico.

Financiamiento: La presente investigación no fue financiada por alguna entidad ni patrocinado.

Conflicto de Intereses: No existen conflicto de intereses de parte de los autores de la investigación.

profesionales sobre las credenciales necesarias para realizar evaluaciones psicológicas en Puerto Rico. Autor. https://docplayer.es/13102839-asociacion-de-psicologia-de-puerto-rico-p-o-box-363435san-juan-pr-00936-3435-787-751-7100-fax-787-758-6467-info-asppr-net.html

Asociación de Psicología Escolar de Puerto Rico. (2020). Pronunciamiento oficial sobre servicios de evaluación psicológica en condiciones de distanciamiento social: recomendaciones generales para administradores de escuelas, proveedores de servicios y familias. Autor. 
http://apeppr.com/resources/documents/pronunciamiento\%20oficial\%20sobre $\% 20$ procesos $\% 20$ de $\% 20$ evaluación $\% 20$ en $\% 20$ tiempos $\% 20$ de $\% 20$ distanciamiento\%20social-\%20recomendaciones\%20generales.pdf

Ball, C., \& Puffett, A. (1998). The assessment of cognitive function in the elderly using videoconferencing. Journal of Telemedicine and Telecare, 4(1), 36-38. https://doi.org/10.1258/1357633981931362

Ball, C., Tyrrell, J., \& Long, C. (1999). Scoring written material from the Mini-Mental State Examination: A comparison of face-toface, fax and video-linked scoring. Journal of Telemedicine and Telecare, 5(4), 253-256. https://doi.org/10.1258/1357633991933819

Barak, A., \& Buchanan, T. (2004). Internet-based psychological testing and assessment. In R. Kraus, G. Stricker, \& C. Speyer (Eds.), Online counseling: A handbook for mental health professionals (pp. 4217-4239). Elsevier Academic Press.

Brearly, T.W., Shura, R.D., Martindale, S.L., Lazowski, R.A., Luxton, D.D., Shenal, B.V., \& Rowland, J.A. (2017). Neuropsychological test administration by videoconference: A systematic review and meta-analysis. Neuropsychology Review, 27(2), 174186. https://doi.org/10.1007/s11065-017-9349-1

Byers, A. P. (1981). Psychological evaluation by means of an online computer. Behavior Research Methods \& Instrumentation, 13, 585587. https://doi.org/10.3758/BF03202069

Camara de Reprrsentantes de Puerto Rico. (2020). Resolución Conjunta de la Cámara, Núm. 641. Autor. http://www.tucamarapr.org/dnncamara/Documents/Measures/fdfdb72d-232a-43db-afe7ee81b1f5e285.pdf?fbclid=IwAR2kVvLxs-o4vMIG3gQN-NgIWUERucBKdoRQUcXITV1T0yhv8_TecwEJH7w

Cullum, C. M., Weiner, M. F., Gehrmann, H. R., \& Hynan, L. S. (2006). Feasibility of telecognitive assessment in dementia. Assessment, 13(4), 385-390. https://doi.org/10.1177/1073191106289065

Glaze, R., \& Cox, J. L. (1991). Validation of a computerized version of the 10-item (self-rating) Edinburgh postnatal depression scale. Journal of Affective Disorders, 22(1-2), 73-77. https://doi.org/10.1016/0165-0327(91)90086-8

Health Insurance Portability and Accountability Act (HIPAA), Pub., L, 104-191. (1996). www.lexjuris.com/lexmate/salud/lexleyhippaespanol.htm

Junta Examinadora de Psicólogos de Puerto Rico. (1992). Código de Ética. Autor.

Junta Examinadora de Psicólogos de Puerto Rico. (2020). Uso de la tecnología para ofrecer servicios psicológicos durante la pandemia de COVID-19. Autor.

Kirkwood, K. T., Peck, D. F., \& Bennie, L. (2000). The consistency of neuropsychological assessments performed via telecommunication and face to face. Journal of Telemedicine and Telecare, 6(3), 147-151. https://doi.org/10.1258/1357633001935239

Ley Núm. 169 de 1 de agosto de 2018. Ley para el Uso de la Telemedicina en Puerto Rico. http:/www.lexjuris.com/lexlex/Leyes2018/lex12018168.htm
Ley Núm. 48 de 29 de abril de 2020. Ley para Regular la Ciberterapia en Puerto Rico. http://www.lexjuris.com/lexlex/Leyes2020/lex12020048.htm

Ley Núm. 96 de 4 de junio de 1983, según enmendada. Ley para Reglamentar el Ejercicio de la Profesión de la Psicología en Puerto Rico. http://www.lexjuris.com/lexmate/profesiones/lex103.htm

Luxton, D. D., Pruitt, L. D., \& Osenbach, J. E. (2014). Best practices for remote psychological assessment via telehealth technologies. Professional Psychology: Research and Practice, 45(1), 27-35. https://doi.org/10.1037/a0034547

Maheu, M. M., \& Gordon, B. L. (2000). Counseling and therapy on the Internet. Professional Psychology: Research and Practice, 31(5), 484-489. https://doi.org/10.1037/0735-7028.31.5.484

Naglieri, J. A., Drasgow, F., Schmit, M., Handler, L., Prifitera, A., Margolis, A., \& Velasquez, R. (2004). Psychological Testing on the Internet: New Problems, Old Issues. American Psychologist, 59(3), 150-162. https://doi.org/10.1037/0003-066X.59.3.150

R-PAS: Recommendations concerning remote administration of the Rorschach (April, 2020) https://r-pas.org/docs/remote\%20administration\%20of\%20the\%20rorschach.pdf

Raiford, Susan E. (2017). “Intelligently and Intuitively Incorporating the Integrated into WISC-V Interpretation." Essentials of WISC-V Integrated Assessment, edited by Alan S Kaufman and Nadeen L Kaufman, John Wiley \& Sons, Hoboken, NJ, 2017, pp. 123-178.

Reynolds, C. R. (2014). Reynolds Adaptable Intelligence Test: Professional Manual. PAR.

Reynolds, C. R., \& Kamphaus, R. W. (2015). Reynolds Intellectual Assessment Scales, Second. Edition and the Reynolds Intellectual Screening Test (2nd ed.). PAR.

Sharp, J. (2020b). Telehealth Assessment of Children [Webinar]. Nebraska Psychological Association.

Sharp, J. (Host). (2020a, April 3). Remote Administration of the WISC-V and KTEA III with Dr. Susie Raiford (112) [Audio podcast episode]. In The Testing Psychologist Podcast. http://www.thetestingpsychologist.com/remote-administration-of-the-wiscv-ktea-iii-w-dr-susie-raiford/.

Stolwky, R., Hammers, D. B., Harder, L. \& Cullum, C. M. (2020, April 2). Teleneuropsychology (TeleNP) in Response to COVID-19: Practical Guidelines to Balancing Validity Concerns with Clinical Need. [Power Point Presentation]. https://www.the-ins.org/files/webinars/20200402_covid19/INS_COVID19_Webinar-20200402.pdf.

Wright, A. J. (2018). Equivalence of Remote, Online Administration and Traditional, Face-to-Face Administration of the Reynolds Intellectual Assessment Scales-Second edition (Online white paper). Presence Learning.

Wright, A. J. (2020). Learn more about Ethical and Practical Considerations for Psychological Tele-Assessment During COVID-19 [Webinar]. Riverside Insights. https://register.gotowebinar.com/recording/7314002332415112450. 